\title{
Article
}

\section{Constraints on General Relativity Geodesics by a Covariant Geometric Uncertainty Principle}

\author{
David Escors ${ }^{1 *}$ and Grazyna Kochan ${ }^{2}$ \\ 1 Fundacion Miguel Servet. UPNA; descorsm@navarra.es \\ 2 Fundacion Miguel Servet. UPNA. grazyna.kochan@navarra.es \\ * Correspondence: descorsm@navarra.es
}

\begin{abstract}
General relativity is a theory for gravitation based on Riemannian geometry, difficult to compatibilize with quantum mechanics. This is evident in relativistic problems in which quantum effects cannot be discarded. For example in quantum gravity, gravitation of zero-point energy or events close to a black hole singularity. Here, we set up a mathematical model to select general relativity geodesics according to compatibility with the uncertainty principle. To achieve this, we derived a geometric expression of the uncertainty principle (GeUP). This formulation identified proper space-time length with Planck length by a geodesic-derived scalar. GeUP imposed a minimum allowed value for the interval of proper space-time which depended on the particular spacetime geometry. GeUP forced the introduction of a "zero-point" curvature perturbation over flat Minkowski space, caused exclusively by quantum uncertainty but not to gravitation. When applied to the Schwarzschild metric and choosing radial-dependent geodesics, our mathematical model identified a particle exclusion zone close to the singularity, similar to calculations by loop quantum gravity. For a 2 black hole merger, this exclusion zone was shown to have a radius that cannot go below a value proportional to the energy/mass of the incoming black hole multiplied by Planck length.
\end{abstract}

Keywords: General relativity; Uncertainty principle; Geodesics; Black hole singularity; zero-point energy

\section{Introduction}

General Relativity (GR) describes gravitation as a dynamical space-time geometry in a pseudo-Riemannian manifold shaped by energy-momentum densities [1]. Its mathematical framework is highly consistent and valid in any reference frame through tensor equations. However, GR is difficult to compatibilize with the mathematics of quantum mechanics. Hence, how the geometry of gravitation and quantum processes interact is still unclear, although some theories such as loop quantum gravity can integrate their methodologies [2].

Some GR solutions involve regions of space-time with infinite curvature, such as black hole singularities [3-5]. As a geometry, infinite curvature is not in itself problematic or unphysical, and a black hole singularity represents just a shape of space-time. However, a black hole singularity creates a fundamental physical problem because it would not be possible to trace back the history of any particle that ends up in it. This irreversible process contributes to the black hole information paradox [6].

It could be nevertheless argued that particles in a black hole singularity do differ in proper time. However, proper time tends to infinity closer to the singularity, and it is not defined right at the singularity. Therefore, information is irreversibly lost if singularities are not restricted by other fundamental processes. As particles get close to a black hole 
singularity, quantum physics can provide a solution. Heisenberg's uncertainty principle [7] has been applied in loop quantum gravity for this purpose as a source of a repulsion force that prevents particles from reaching the singularity [8]. The matter repelled from it would form a so-called "Planck star". An "equivalent" concept is used in string theory with the "fuzzball" structure [9].

The non-linearity of GR mathematics is also in sharp contrast with the linearity of quantum mechanics. This also introduces complications of its own. For example, there are difficulties in co-variant formulations of a general uncertainty principle [10]. As energy is also a source of gravitation in GR, it is difficult to understand how quantum mechanical processes such as zero-point energy fluctuations influence the space-time geometry. This difficulty complicates quantum gravitation theories.

Here we develop a co-variant relativistic geometric formulation of the uncertainty principle within the mathematical framework of GR. This resulted in a geometric principle of uncertainty (GeUP) for particles in geodesics. This was achieved by generating a relativistic tensor expression for the inequalities of the classical uncertainty principle. This tensor equation was then converted in an inequality containing only geometric terms. This inequality identified Planck length with the uncertainty of relativistic proper space-time distance through a scalar derived from geodesic geometries. Then GeUP was applied to a particle in Minkowski space in the absence of gravitation. The imposition of GeUP required the need for a "zero-point" curvature perturbation. GeUP was finally applied to a particle in the Schwarzschild metric, with special focus on the singularity and event horizon. An exclusion zone from the singularity was obtained as a solution. This exclusion zone provided a minimum threshold value around the singularity of comparable order to the estimated radius for Planck stars.

\section{Derivation of a relativistic tensor expression for the uncertainty principle inequali- ties}

For simplicity, $\mathrm{c}$ and the particle mass were both set to 1 . Tensor notation was used throughout the paper, which includes representation of generalized contravariant coordinates as $\mathrm{X}^{\mu}$. For clarity, the temporal coordinate $\mathrm{X}^{0}$ was represented as " $\mathrm{t}$ " in some specific cases.

The classical uncertainty principle is represented by two separate inequalities.

$$
|\Delta \vec{p}||\Delta \vec{x}| \geq \frac{\hbar}{2} \quad ;|\Delta E|\left|\Delta x^{0}\right| \geq \frac{\hbar}{2}
$$

Small " $\mathrm{p}$ " represents non-relativistic momentum, parametrized by coordinate time. These two inequalities can be written in tensor notation, following these identities:

$$
|\Delta \vec{p}||\Delta \vec{x}|=\left|\sqrt{\Delta p^{m} \Delta x_{m} \Delta p_{m} \Delta x^{m}}\right|=\left|\Delta p_{m} \Delta x^{m}\right|
$$

\section{$\Delta \boldsymbol{E} \equiv \Delta \boldsymbol{P}_{0}$}

In units of c set to 1, energy can be identified with the temporal component of the relativistic 4-momentum vector, which is parametrized by proper time " $\boldsymbol{\tau}$ ". From now on, the relativistic momentum will be represented by capital " $\mathrm{P}$ ". Inequalities 1 then take the following form in tensor notation:

$$
\left|\Delta p_{m} \Delta X^{m}\right| \geq \frac{\hbar}{2} ;\left|\Delta P_{0} \Delta X^{0}\right| \geq \frac{\hbar}{2}
$$




$$
\mathrm{m} \in\{1,2,3\}
$$

To convert non-relativistic momentum to its relativistic counterpart parametrized by proper time, the gamma factor has to be introduced. The same correction was included in the energy-time inequality so that both can be merged.

$$
\left|\frac{1}{\gamma} \Delta P_{m} \Delta X^{m}\right| \geq \frac{\hbar}{2} ;\left|\frac{1}{\gamma} \Delta P_{0} \Delta X^{0}\right| \geq \frac{\hbar}{2 \gamma}
$$

Adding inequalities 3 we obtain after some re-arrangements:

$$
\left|\Delta \boldsymbol{P}_{m} \Delta X^{m}+\Delta P_{0} \Delta X^{0}\right| \geq(1+\gamma) \frac{\hbar}{2}
$$

For non-relativistic particles, the gamma factor is generally 1, which will lead to a standard general form of the non-relativistic uncertainty principle:

$$
\left|\Delta \boldsymbol{P}_{m} \Delta \boldsymbol{X}^{m}+\Delta \boldsymbol{P}_{0} \Delta \boldsymbol{X}^{\mathbf{0}}\right| \geq \hbar
$$

The merged inequality can then be stated as a contraction of differential changes in 4-momentum and 4-position vectors as follows:

$$
\begin{gathered}
\left|\boldsymbol{d} \boldsymbol{P}^{\mu} \boldsymbol{d} \boldsymbol{x}_{\boldsymbol{\mu}}\right| \geq \frac{\hbar}{\mathbf{2}} \\
\mu \in\{0,1,2,3\}
\end{gathered}
$$

For simplification, the relativistic correction term in inequality 4 was removed as it can be easily incorporated when needed. We then re-expressed the inequality in terms of Planck length:

$$
\left|d P^{\mu} d x_{\mu}\right| \geq \frac{\ell_{p}^{2}}{2 G}
$$

The inequality was then further parametrized as a function of differential changes in proper time as follows:

$$
\left|\frac{d P^{\mu}}{d \tau} d \tau \frac{d x_{\mu}}{d \tau} d \tau\right| \geq \frac{\ell_{p}^{2}}{2 G}
$$

This allows de re-formulation in terms of the co-variant proper velocity components $\left(\mathrm{U}_{\mu}\right)$, the change in 4-momentum with proper time, and the introduction of the invariant quadratic form of proper space-time distance. It has to be noted that this operation makes the inequality undefined for null proper time.

$$
\left|\frac{d P^{\mu}}{d \tau} U_{\mu} d \tau^{2}\right| \geq \frac{\ell_{p}^{2}}{2 G}
$$

This expression has to be interpreted as an inequality between the uncertainties of proper space-time length and 4-momentum change. In the non-quantum limit where Planck length is considered 0 , equation 9 eliminates this uncertainty by being equated to 0 . In this case, proper space-time and 4-momentum change ("proper force") can be known with infinite precision. 


\section{Derivation of a relativistic geometric form of the uncertainty principle}

In the framework of GR, space-time geodesic trajectories for particles are expressed in terms of proper velocity components $\left(\mathrm{U}^{\mu}\right)$, which can be identified with proper momentum considering a unit mass in the equations:

$$
\frac{d U^{\mu}}{d \tau}=-\Gamma_{\alpha \beta}^{\mu} U^{\alpha} U^{\beta} \equiv \frac{d P^{\mu}}{d \tau}
$$

The expression for the change of proper momentum above can be substituted in inequality 9:

$$
\left|-\Gamma_{\alpha \beta}^{\mu} U^{\alpha} U^{\beta} U_{\mu} d \tau^{2}\right| \geq \frac{\ell_{p}^{2}}{2 G}
$$

This resulting expression is fully dependent on the specific geometry of relativistic space-time by Christoffel connectors contracted with proper velocities. These contractions allow inequality 10 to be further re-arranged as the product of two scalars by defining a "Geometric scalar", or Ggeo:

$$
\begin{aligned}
& G_{g e o} \equiv-2 G U_{\mu} \Gamma_{\alpha \beta}^{\mu} U^{\alpha} U^{\beta} \\
& \left|G_{g e o} d \tau^{2}\right| \geq \ell_{p}^{2}
\end{aligned}
$$

This relationship makes a direct identification between the uncertainty in proper space-time length with Planck length, by a scale factor that depends on the geodesic. This inequality imposes a minimum degree of uncertainty in proper space-time that depends on the geometry. Therefore, proper time represented in units of Planck length has as the lowest limit the inverse of the absolute value of the geometric scalar (without the relativistic correction as in inequality 4):

$$
\left|\frac{d \tau^{2}}{\ell_{p}^{2}}\right| \geq\left|G_{g e o}^{-1}\right|
$$

This inequality represents the relationship between proper space-time and Planck length which is at the core of GeUP.

\section{Application of the geometric uncertainty principle to geodesics in flat Minkowski space}

GeUP was applied to flat Minkowski space for a test particle at rest. As an immediate consequence, inequality 11 cannot be met because the geodesic scalar is 0 by the null Christoffel connectors.

$$
\begin{gathered}
\Gamma_{\alpha \beta}^{\mu}=0 \rightarrow G_{g e o}=0 \\
\left|G_{g e o} d \tau^{2}\right|=0 \geq l_{p}^{2} ?
\end{gathered}
$$

Flat Minkowski space leads to a contradiction (inequality 13) with the exception of the non-quantum limit in which Planck length is considered 0. To meet the GeUP condition, Minkowski space has to deviate from flat space. To obtain general allowed solutions for metric tensors is challenging because the perturbation can be a function of all the coordinates, with cross-terms and uncertainties involving all coordinates. Hence, to study 
the main consequences, here we used a weak field approximation approach, adding a differential perturbation to the Minkowski metric represented as eta:

$$
g_{\mu v}=\eta_{\mu v}+\varepsilon_{\mu v}
$$

In our example, the perturbation depends solely on the temporal component to fulfil conditions of spatial homogeneity and isometry. In this special case, Planck constant will be explicitly used in equation 11 . For a particle at rest only the temporal component of its proper velocity will be non-zero. Likewise, only coordinate time will contribute to proper time. The inequality takes the following form:

$$
\left|-2 U_{0} \Gamma_{00}^{0} U^{0} U^{0}(-1-\varepsilon) d t^{2}\right| \geq \hbar
$$

The calculation of the Christoffel symbol is straightforward because only the timetime component of the metric tensor has a dependency in the time coordinate but not in spatial coordinates.

$$
\Gamma_{00}^{0}=\frac{1}{2} g^{00}\left(\partial_{0} g_{00}\right)=\frac{-1}{2(1+\varepsilon)} \partial_{0}(-1-\varepsilon)=\frac{\partial_{0} \varepsilon}{2(1+\varepsilon)}
$$

After introducing the term (16), the inequality 15 can be solved as follows:

$$
\begin{aligned}
& \quad\left|-2 U_{0} U^{0} U^{0} \frac{\partial_{0} \varepsilon}{2(1+\varepsilon)}(-1-\varepsilon) d t^{2}\right| \geq \hbar \\
& \left|-U^{0} \partial_{0} \varepsilon d t^{2}\right| \geq \hbar
\end{aligned}
$$

This inequality can be re-expressed by simplifying the derivative of the perturbation field by multiplication with $d t$, and including explicitly the mass of the particle which converts proper velocity to the temporal component of the 4-momentum vector:

$$
\left|P^{0} d \varepsilon \quad d t\right| \geq \hbar
$$

This expression is equivalent to the classical uncertainty principle for time-energy. The time component of the 4-momentum of the particle can best be expressed in terms of the particle's energy. In this expression the relativistic factor omitted in inequality 4 will also be re-introduced with a value of 2 for a rest, non-relativistic particle, leading to:

$$
|E d \varepsilon d t| \geq 2 \hbar ;|d \varepsilon| \geq\left|\frac{2 \hbar}{E d t}\right|
$$

E corresponds in this particular example to the mass-energy of the particle because the particle at rest does not have kinetic energy. We can express the differentials as intervals to represent uncertainties, and express the inequality as an equation for the lowest bound value that would agree with GeUP.

$$
\varepsilon=\varepsilon_{0}+\frac{2 \hbar}{E \Delta t}
$$

The initial value for the perturbation field can be chosen as 0 , which would correspond to no correction over the Minkowski metric. This leads to an expression for the components of the Minkowski metric as follows: 


$$
\begin{aligned}
& g_{00}=-1-\frac{2 \hbar}{E \Delta t} \quad ; g_{m m}=1+\frac{2 \hbar}{E \Delta t} \\
& m \in\{1,2,3\}
\end{aligned}
$$

This solution resembles a zero-point energy in a way that it arises from the necessity to fulfil the uncertainty principle, and it is significant under very small intervals of time. The non-quantum limits correspond to massive particles and events over large intervals of time, which make the perturbation go to 0 and recover the Minkowski metric. This perturbation is not caused by gravitation from the mass of the particle, but from the GeUP principle.

\section{Application of the geometric uncertainty principle to Schwarzschild's metric}

GeUP was applied to Schwarzschild's metric in spherical coordinates, with a $\{-++$ $+\}$ metric signature. The black hole singularity is present at the 0 radial position. Right at the singularity, all spatial coordinates for the particle are known with absolute certainty, with the exception of the temporal component. To find out if this condition is sufficient to overcome the principle of uncertainty, inequality 11 was applied. Absolute certainty in position corresponds to null proper velocities of spatial components. Only the temporal component of the proper velocity plays a contribution, whereby only a Christoffel connector survives in the geodesic scalar. However, this is a null Christoffel connector in the Schwarzschild metric:

$$
\begin{aligned}
& d R=d \theta=d \varphi=0 ; \\
& \left|-2 G U_{0} \Gamma_{00}^{0} U^{0} U^{0} d \tau^{2}\right| \geq \ell_{p}^{2} ; 0 \geq \ell_{p}^{2} ?
\end{aligned}
$$

The result is a contradiction. Geodesics with known spatial coordinates but undefined temporal coordinates still contradict GeUP. Therefore, a particle cannot be placed in the point singularity without violating the uncertainty principle.

Solutions that satisfy the inequality 11 in this metric are highly complex and nontrivial, involving the interplay between all the coordinates through Christoffel connectors, and uncertainties in all coordinates. In this paper, we chose geodesics with constant angular and temporal coordinates, but with varying radial position. This constitutes a simplified "snapshot" in coordinate time, allowing only uncertainty in the radial position. Hence, this selection of geodesics will also make the proper velocity of the particle to have only a radial component as shown below:

$$
d t=d \theta=d \varphi=0 \rightarrow U^{0}=U^{\theta}=U^{\varphi}=0
$$

For simplification, a test unit mass will be considered and $\mathrm{c}$ will be set to 1 . The selection of these initial conditions simplifies the calculation of the geodesic scalar as only the radial term is non-zero, giving a result of:

$$
\begin{aligned}
G_{\text {geo }} & =-2 G U_{R} \Gamma_{R R}^{R} U^{R} U^{R}=-2 G U_{R} U^{R} \Gamma_{R R}^{R} U^{R}=2 G \Gamma_{R R}^{R} U^{R} \\
G_{\text {geo }} & =\frac{G R_{S} U^{R}}{R\left(R-R_{S}\right)}
\end{aligned}
$$

And proper time is, 


$$
d \tau^{2}=\frac{R}{R-R_{S}} d R^{2}
$$

$\mathrm{R}_{\mathrm{s}}$ indicates Schwarzschild's radius. With the terms in equations 23 and 24, the inequality 11 can be then solved leading to:

$$
d R^{2} \geq\left|\left(R-R_{S}\right)^{2} \frac{\ell_{p}^{2}}{G R_{S} U^{R}}\right|
$$

This inequality solves the uncertainty in radial position squared as a function of radial distance. This equation can be re-written in a more concise form and also re-incorporating the particle mass " $\mathrm{m}$ " into the proper velocity, with M representing the mass generating the gravitational field:

$$
d R^{2} \geq\left|\frac{2 M}{P^{R}}\left(\frac{R}{R_{s}}-1\right)^{2} \ell_{p}^{2}\right|
$$

\subsection{Uncertainty in the interior of the black hole.}

Inequality 26 sets the lower threshold for radial uncertainty to a term that is proportional to Planck length and multiplied by a function of the radial position. Considering the lowest allowed uncertainty condition in the geodesics, we reach two conclusions. First, it is defined at the singularity $(R=0)$. Second, as the particle approaches the singularity, the uncertainty in radial position of the particle increases. A relative coefficient of threshold uncertainty can then be calculated as follows:

$$
C U=\frac{d R}{R}= \pm \sqrt{\frac{2 M}{P^{R}}}\left(\frac{1}{R_{s}}-\frac{1}{R}\right) \ell_{p}
$$

The coefficient of uncertainty in radial position within the black hole exponentially grows as the particle approaches the singularity (Figure 1), tending to infinity.

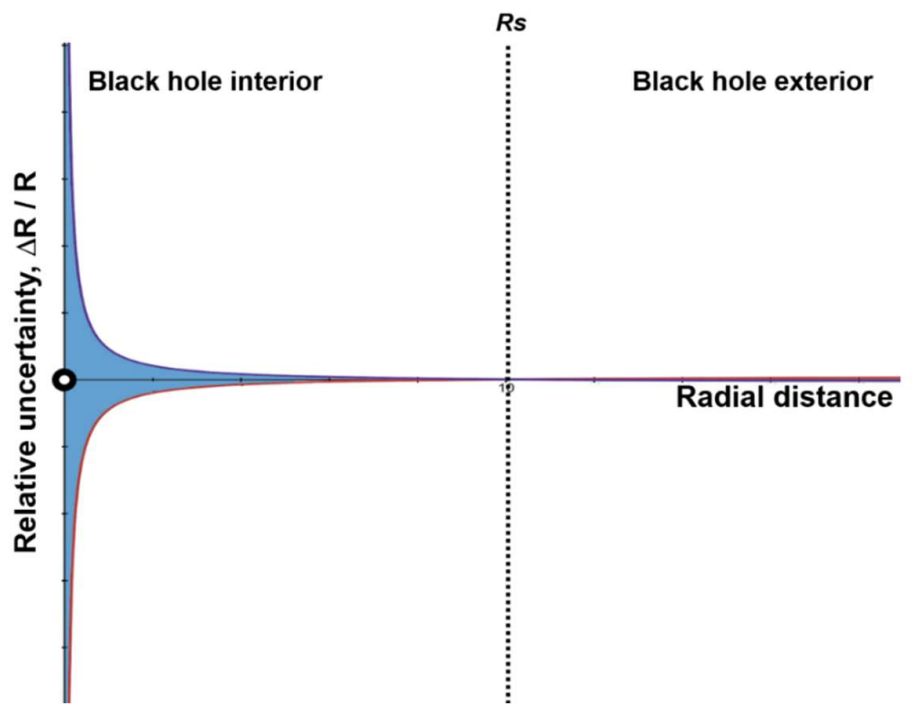

Figure 1. Black hole exclusion zone. The figure represents the function from equation 27 with relative values for the radial distance and coefficients to help visualization. The relative uncertainty in 
radial position with radial distance is plotted, dividing the phase space in two sections. The blue section identifies geodesics that violate the GeUP (exclusion zone). Relative uncertainties for allowed geodesics grow exponentially towards the singularity (open dot at $\mathrm{R}=0$ ). The zones in white within the black hole interior or its exterior indicate allowed geodesics in which particles can be found with at least the relative uncertainties indicated by the curves.

Importantly, inequality 26 does have a minimum allowed threshold value for uncertainty right in the singularity:

$$
d R^{2}\left(R_{0}\right) \geq \frac{2 M}{P^{R}} \ell_{p}^{2}
$$

It is important to note that the uncertainty in position is proportional to Planck length, but further modified by the momentum of particles. Close to the singularity there is an "exclusion zone" given by inequality 28 , below which no particle can be found with definite position (Figure 1). In addition, most particles close to the singularity will be relativistic as well, so the relativistic correction needs to be specified in inequality 28 , leaving:

$$
d R^{2}\left(R_{0}\right) \geq\left(1+\frac{E}{m}\right) \frac{2 M}{P^{R}} \ell_{p}^{2}
$$

The uncertainty in radial position of particles reduces as the radial distance approaches Schwarzschild's radius (Figure 1 and inequality 26). From this place onwards, the uncertainty in position steadily increases as the radial position goes to infinity. Taking the limit of inequality 26 to infinity brings $d R$ to infinity, although this mathematical limit is unphysical. The radial distance that cancels out Planck length in inequality 26 is of the order of 3 light-years, which could be considered a physical infinity in the Schwarzschild metric. Indeed, the coefficient of uncertainty at this position is of the order of $10^{-29}$.

Therefore, at radial distances much larger than Planck distance, this latter value can be regarded as 0 , reaching the classical and relativistic limits where the particle can have both infinitely precise momentum and position.

\subsection{Merger of two black holes}

Within the limits of our approximation to geodesics with only radial dependence, we can evaluate an incoming particle with a mass comparable to the black hole's mass at near relativistic velocities. These conditions can be then applied to inequality 26 in units of c set to 1, giving:

$$
\begin{aligned}
& P^{R} \approx M ; R=0 \\
& d R^{2} \geq 2 \ell_{p}^{2}
\end{aligned}
$$

Hence, the singularity exclusion radius cannot get lower than Planck length. However, the merging of black holes is a highly energetic process, and the relativistic correction cannot be discarded. This will lead to the final result in units of $\mathrm{c}$ set to 1 as:

$$
d R^{2} \geq\left(1+\frac{E}{M}\right) 2 \ell_{p}^{2} \approx \frac{2 E}{M} \ell_{p}^{2}
$$

The exclusion value is still proportional to the Planck length, but it is dependent on the ratio of between the total energy of the incoming black hole and its mass-energy. As this ratio will always be higher than 1 , in no case the radius of the exclusion zone will get smaller than the Planck length. Following the merger, the singularity exclusion zone will 
increase to accommodate the additional mass together with the new Schwarzschild radius, incorporating its information as a growth in the radius of the exclusion zone, leaving inequality 26 as:

$$
d R^{2} \geq\left|\frac{4 M}{P^{R}}\left(\frac{R}{R_{s}}-1\right)^{2} l_{p}^{2}\right|
$$

Therefore, the uncertainty principle could be considered the source of a repulsion force which becomes very large close to the singularity. In this paper, we associate this force to the geometry of the geodesics into a geometric uncertainty principle.

\section{Discussion}

Here we have expressed the principle of uncertainty in tensor form, which has allowed its reformulation in terms of geometric parameters consistent with both GR and quantum mechanics. Indeed, using our mathematical model we found that there is a limit for the length of relativistic space-time distance restricted by Planck lengths. It is important to remark that this observation agrees with theoretical calculations in loop quantum gravity models [8]. This relationship is also "fuzzy", relating the uncertainty of proper space-time distance with Planck length through a scaling factor coming from the specific geometry of the GR geodesics.

To test GeUP and understand its immediate consequences, we applied the equation to the simplest case: A particle at rest in Minkowski flat space. GeUP forced the need of introducing a "zero-point curvature" field so that geodesics comply with the uncertainty principle. For computational simplicity, and to comply with isometry and homogeneity, we introduced a weak-field perturbation that was dependent only on the time coordinate. We found a similar result to the classical energy-time uncertainty in quantum mechanics: Events taking place in small intervals of time are linked to large energy fluctuations. GeUP forced the perturbation to increase inversely proportional to the time length interval. In the classical/relativistic limit of long intervals of time, the correction to the metric can be discarded. Interestingly, it also depended on the mass of the particle. The larger the mass, the weaker the perturbation. This result highlighted that the "zero-point curvature" is exclusively a quantum effect not caused by gravitation. This can be significant for scenarios of quantum gravity, for which gravitational-like effects may appear as the result of GeUP which could be superimposed on effects by standard gravitation. It is interesting to remark that propagation of gravitational waves as a solution in GR [11,12] provides weak field perturbations that comply with GeUP.

Then we tested GeUP in a more complex scenario. We found that applying GeUP to the Schwarzschild's metric required a computational power beyond the scope of the current paper. For a full solution, allowed geodesics have to be found in a phase space with 4 coordinate positions, 4 coordinate uncertainties, and multiple cross-terms. Therefore, to draw physically relevant solutions, we first demonstrated that placing a particle right in the black hole singularity violates GeUP. This is important, because particles could be theoretically singled-out on the basis of their different coordinate times at the singularity. As such, particles in the singularity could comply with a general uncertainty principle [10] because they would not be in the same states. But interestingly, GeUP selects null Christoffel connectors in the Schwarzschild's metric right in the singularity. This condition nullifies inequality 22, leading to a contradiction. Once this point was clear, we selected geodesics which only depended in radial position. We have to stress that this system loses the dynamics of the particles, but provides important insight. Application of GeUP to the black hole immediately identified a region close to the singularity below which there 
would be a violation of GeUP for particles with defined position. This region consists of a black hole exclusion zone, and the minimum radius was found to be proportional to Planck length. Nevertheless, our inequalities do not necessarily forbid particles to enter this zone. The inequalities shown here have to be interpreted as uncertainties in proper position, whereby particles can be more or less de-localized. In this regard, the exclusion zone can be thought of a place with very high uncertainty in position, with "fuzzy" particles which may be equivalent to the "fuzzballs" found in string theory [9].

From a more classical quantum mechanics point of view, particles close to the singularity will have an uncertainty in position so large that they would appear to be repelled from the singularity. Our results are similar to those in loop quantum gravity, which showed that matter would be distributed spherically with a radius several orders of magnitude higher than Planck length $[2,8]$. This repulsion "uncertainty force" avoids singlepoint singularities leading to the theoretical formation of a Planck star. Hence, the Planck star would be made of the space taken by all GeUP-allowed geodesics found in the current model. Carrying out calculations on the minimum radial threshold for the exclusion zone corresponding to a stellar mass black hole, inequality 28 gives a rough approximate value (without adding specific corrections) on the order of $10^{-16} \mathrm{~cm}$. A rough estimation on the diameter of a Planck star by loop quantum gravity gives a value of about $10^{-10} \mathrm{~cm}$ [8]. Although there is a difference of over 6 orders of magnitude between both calculations, this difference can be accounted by further fine tuning of the adequate corrections and parameters to the equations both here and those from loop quantum gravity. Nevertheless, both calculations provide sizes larger than Planck length by several orders of magnitude.

We also tested our model in a highly energetic process, such as a 2-black hole merger. Again, it has to be remarked that we lost the dynamics by our choice of geodesics. Nevertheless, important conclusions could be obtained. First, in no case the radius of the exclusion zone will go below Planck length. Second, after the merger there surface of the exclusion zone will grow, allowing the accommodation of the extra information carried by the incoming black hole.

Summarizing, we have derived a geometric formulation for the uncertainty principle that selects geodesics complying with this fundamental quantum law. The application of this restriction will be helpful in general relativity problems for which quantum effects cannot be discarded.

Author Contributions: Conceptualization, D.E. and G.K.; Methodology, D.E; Resources, D.E. and G.K. All authors have read and agreed to the published version of the manuscript."

Acknowledgments: The authors thank Dr Funfelinski for critical insight.

Funding: D.E. is funded by a Miguel Servet Fellowship (ISCIII, Spain, Ref CP12/03114).

Conflicts of Interest: The authors declare no conflict of interest.

\section{References}

1. $\quad$ Einstein, A. Grundlage der allgemeinen Relativitätstheorie. Annalen der Physik 1916, 49, 769-822.

2. Rovelli, C. Loop Quantum Gravity. LivingRev.Rel. 1998, 1, doi:10.12942/lrr-1998-1.

3. Schwarzschild, K. On the gravitational field of a mass point according to Einstein's theory. Sitzungsber.Preuss.Akad.Wiss.Berlin (Math.Phys.) 1916, 1916, 189-196.

4. Teukolsky, S.A. The Kerr metric. Class. Quantum Grav. 2015, 32, 124006.

5. Townsend, P.K. Black holes. arXiv:gr-qc/9707012 1997.

6. Dai, X. The Black Hole Paradoxes and Possible Solutions. J. Phys.: Conf. Ser. 2020, 1634, 012088.

7. Ozawa, M. Heisenberg's original derivation of the uncertainty principle and its universally valid reformulations. arXiv:1507.02010 215. 
8. Rovelli, C.; Vidotto, F. Planck stars. International Journal of Modern Physics D. 2014, 23, 1442026.

9. Mathur, S.D. The Fuzzball proposal for black holes: An Elementary review. Fortsch.Phys. 2005, 53, $793-827$.

10. Todorinov, V.; Bosso, P.; Das, S. Relativistic generalized uncertainty principle. Annals Phys. 2019, 405, 92-100, doi:10.1016/j.aop.2019.03.014.

11. Abbott, B.P.; Abbott, R.; Abbott, T.D.; Acernese, F.; Ackley, K.; Adams, C.; Adams, T.; Addesso, P.; Adhikari, R.X.; Adya, V.B. Gravitational Waves and Gamma-Rays from a Binary Neutron Star Merger: GW170817 and GRB 170817A. ApJ 2017, 848, L13.

12. Cornish, N.; Blas, D.; Nardini, G. Bounding the Speed of Gravity with Gravitational Wave Observations. Phys. Rev. Lett. 2017, 119, 161102. 